\title{
Interkulturelles Übersetzen und die Betreuung «Unsichtbarer»
}

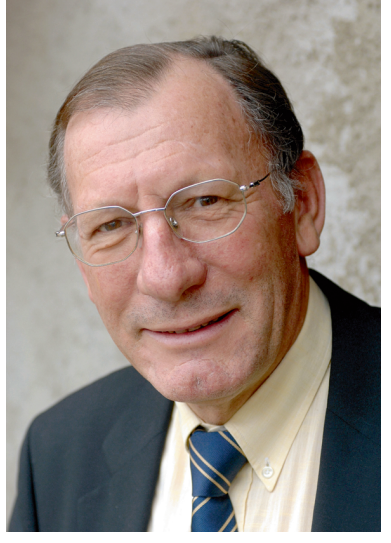

Jean Martin*

* PD Dr. med. Jean Martin, Mitglied der Redaktion, ist ehemaliger Kantonsarzt im Kanton Waadt und Mitglied der Nationalen Ethikkommission

1 Joss M. Interkulturelles Übersetzen als Vertrauensgrundlage bei der Behandlung von Patientinnen und Patienten mit Migrationshintergrund. Schweiz Ärztezeitung. 2008;89(8):320-2.

2 Taverna E. Eine Praxis für Unsichtbare. Schweiz Ärztezeitung. 2008; 89(7):290-1.
Der kürzlich erschienene Artikel von Monika Joss [1] ist besonders für unseren Berufsstand informativ und aufschlussreich. Ich nehme mir die Freiheit, dies in meiner Funktion als Mitglied des Vorstandes von Appartenances (www. appartenances.ch) zu bemerken. Unsere Organisation bietet seit rund 15 Jahren im Kanton Waadt, in Lausanne, Vevey und Yverdon, eine Reihe von Diensten für Menschen an, die aus anderen Ländern zu uns gekommen sind. Wir decken verschiedene Bereiche ab: psychotherapeutische Unterstützung, Grundausbildung für Erwachsene und berufliche Weiterbildung, Aktionen und Fördermassnahmen, Animationsprogramme und verschiedene Unterstützungsleistungen (Versammlungsräume), Kurse (Französisch, Informatik, Schneidern usw.).

Eine wichtige Aufgabe des Vereins ist die Ausbildung und Bereitstellung von interkulturellen Übersetzerinnen und Übersetzern. Sie werden hauptsächlich in den Bereichen Gesundheit, Bildung und im sozialen Leben gebraucht, und zwar sowohl innerhalb von Appartenances selbst als auch ausserhalb (Spitäler, Schulen, Sozialdienste). Im letzten Jahr haben wir 17000 Dolmetscherstunden verzeichnet.

Anlässlich des Jubiläums des 10jährigen Bestehens dieses Dienstes organisierte Appartenances Ende 2007 eine Veranstaltung, in deren Mittelpunkt eine sehr interessante Gesprächsrunde über die verschiedenen Formen des Dolmetschens stand. Vertreten waren eine interkulturelle Übersetzerin, ein Konferenzdolmetscher, eine Mediatorin im Bereich Konfliktmanagement und eine Gebärdensprachdolmetscherin (für gehörlose Menschen). Besonders die Beiträge dieser letzten Dolmetscherin brachten für viele Zuhörer neue Gesichtspunkte. Es wurde deutlich, dass sich zwar je nach Kontext unterschiedliche Fragen und Probleme stellen, letztere aber auch grundlegende gemeinsame Dimensionen haben. So zum Beispiel die Frage des Berufskodex, insbesondere der originalgetreuen Übersetzung bzw. Verdolmetschung, sowie die Frage der Schweigepflicht und der Neutralität.

Das interkulturelle Übersetzen hat sich erst seit kurzem entwickelt. Die schweizerische Interessengemeinschaft Interpret, zu der auch Monika Joss gehört, zählt mittlerweile über 500 ausgebildete Übersetzerinnen und Übersetzer. Ihr Äquivalent auf internationaler Ebene ist die Organisation Critical Link. Im Bereich der
Gesundheitsdienste gehört die Hinzuziehung von Dolmetscherinnen und Dolmetschern heute unverzichtbar zur good medical practice. Dabei muss aber erwähnt werden, dass ihre Kosten derzeit noch viel zuwenig von kollektiven Organen (Krankenkasse, öffentliche Hand) übernommen werden. Appartenances und andere erhalten zwar gewisse Zuschüsse, doch die Erstattung von Übersetzungskosten gerät nach wie vor in Vergessenheit. In manchen Fällen ist der Aufbau einer guten therapeutischen Beziehung ohne Zuhilfenahme eines professionell ausgebildeten Dolmetschers schlicht nicht möglich, und es ist nicht hinzunehmen, dass diese Leistungen nicht finanziert werden. Und dies in Form einer regelmässigen Finanzierung, nicht durch Sonderbewilligungen oder jeweilige Einzelentscheide. Dasselbe gilt im übrigen auch für Gespräche in Schulen mit Eltern von Schülern mit anderer Muttersprache.

In diesem Zusammenhang berichtet mir die zuständige Person bei Appartenances von einem Arzt, der eine Rechnung über die Leistungen einer Dolmetscherin abgelehnt hat. Diese hatte er hinzugezogen, um einer Patientin die lebensbedrohlichen Folgen einer Risikoschwangerschaft zu erklären. Man kann diesen Arzt verstehen ... denn wie sollte man von ihm verlangen, den Rechnungsbetrag persönlich aufzubringen? Daher ist es dringend notwendig, die üblichen Finanzierer $\mathrm{zu}$ sensibilisieren - und weiter $\mathrm{zu}$ überzeugen -, damit diese Leistungen übernommen werden. Diese Deckung muss im Rahmen der Grundversicherung erfolgen, denn diejenigen, die Übersetzungsdienste benötigen, gehören häufig zu den Ärmsten der Gesellschaft.

Bei dieser Gelegenheit möchte ich auch den hochinteressanten Artikel von Erhard Taverna erwähnen, der die Arbeit unseres Kollegen David Winizki und seine Praxis für Menschen aus anderen Kulturen beschreibt, nicht selten «Sanspapiers» [2]. Diese Problematik ist bei Appartenances wohlbekannt. Wichtig ist es, all jenen Öffentlichkeit zu verschaffen, die sich um die «Unsichtbaren» in unserer Gesellschaft kümmern. Menschen, die häufig in schwierigen, prekären Verhältnissen leben und nur allzuoft Opfer von Ausbeutung sind, wie der Artikel deutlich macht. Und abschliessend wünschte ich mir, diesen Artikel auch in französischer Sprache lesen zu können!

Jean Martin 